\section{Long-term outcomes after splenectomy in children with immune thrombocytopenia: an update on the registry data from the Intercontinental Cooperative ITP Study Group}

Immune thrombocytopenic purpura (ITP) is an acquired immune-mediated disease characterized by a decrease in platelet count due to antiplatelet autoantibody-mediated increased platelet destruction and, in some cases, associated impaired platelet production. ${ }^{1}$

There has been a decline in the number of splenectomies performed as a second-line treatment after the introduction of new therapies, such as thrombopoietinreceptor agonists. However, the current availability of long-term data on these new second-line therapeutic options seems to suggest that splenectomy remains the more effective second-line intervention in ITP. Therefore, data on long-term outcomes of splenectomy in children are relevant.

The Splenectomy Registry of the Intercontinental Cooperative ITP Study (ICIS) Group collects information regarding children who underwent splenectomy to treat ITP, including perioperative management and follow-up data. A report of the first 134 patients was published in $2007 .^{3}$ The present study aims to update the findings of the ICIS Splenectomy Registry by analyzing over a decade of new data, with a focus on long-term outcomes of children with primary ITP.

Details of the data collection forms of the Splenectomy Registry have been previously described. ${ }^{3}$ Investigators included patients with ITP in whom splenectomy was planned. For the analysis, we excluded records in which splenectomy was not reported or no platelet counts were available at follow-up. Investigators sought local ethics approval prior to patient entry, according to the regulatory requirements of each institution. Response to splenectomy was classified as a complete response (CR: $\geq 60 \%$ of platelet counts performed $\geq 1$ month post-splenectomy $\geq 100 \times 10^{9} / \mathrm{L}$ ), response (R: $\geq 60 \%$ of platelet counts $\geq 1$ month post-splenectomy $\geq 30 \times 10^{9} / \mathrm{L}$ ), or no response (NR: $<60 \%$ of platelet counts $\geq 1$ month post-splenectomy $\left.\geq 30 \times 10^{9} / \mathrm{L}\right) .{ }^{4}$ At least two counts after the first month post-splenectomy were required for outcome assessment. Refractoriness was defined as either the failure to achieve at least $\mathrm{R}$ or loss of $\mathrm{R}$ after splenectomy + clinically relevant bleeding. ${ }^{5}$

Bleeding events were classified as major and clinically relevant non-major (CRNM) according to current definitions of the International Society on Thrombosis and Haemostasis for patients on anticoagulants. ${ }^{6,7}$

The trajectory of platelet counts over time was investigated using generalized estimating equations (GEE) models with basic splines to accommodate for the non-linear shape of the regression line. Predictors of response to splenectomy were explored using logistic regression analysis. Predictors associated with the outcome at $P \leq 0.20$ in univariable analysis were considered for multivariable modeling. For model performance, simple bootstrap with 200 replications was used to estimate the optimism corrected (internally validated) area under the Receiver Operating Characteristic (ROC) curve. Significance was set at an alpha of 0.05 . Analysis was performed in $\mathrm{R}$.

A total of 267 patients were entered in the Registry between June 1997 and September 2017 by 82 investigators from 63 institutions and 26 countries. Seventeen records were excluded due to incompleteness and 11 due to secondary ITP diagnosis. Hence, 239 patients were included in the analysis. Eighty of those patients (80 of $239,33 \%$ ) came from a single institution (The Hospital for Sick Children [SickKids], Toronto, Canada). Patients at SickKids were identified using the ICD-9-CM and ICD-10-CA codes, which explains the large number of participants identified in this institution.

The median duration of follow-up was 25 months ( $25^{\text {th }}-75^{\text {th }}$ percentile: $7.8-53.4$ months). One hundred and thirty-five patients (135 of $250,54 \%$ ) were females. Median age at the time of ITP diagnosis was 9.2 years ( $25^{\text {th }}-75^{\text {th }}$ percentile: $4.8-13.0$ years).

Splenectomy was performed at a median age of 11.7 years $\left(25^{\text {th }}-75^{\text {th }}\right.$ percentile: $7.9-15.3$ years). Sixty-two of the 239 patients $(26 \%)$ had their splenectomy performed $<1$ year after diagnosis (i.e., before reaching chronic ITP status) and ten of those 62 patients $(16 \%)$ had their splenectomy performed $<3$ months after ITP diagnosis (i.e., before reaching persistent ITP status).

There was one intra-abdominal bleeding event documented during surgery. Eleven patients (11 of 239, 5\%), including the patient with intra-abdominal bleeding, received peri-procedural red blood cell transfusions. Twenty-four patients (24 of $239,10 \%$ ) had fever at a median of 41 hours post-procedure (range: 6-99 hours). No deaths or episodes of sepsis in the immediate postoperatory period were recorded.

One-hundred and eighty-six patients were followed up for $>6$ months. No major bleeding events were reported on those patients. Sixteen patients (16 of 184, 8.7\%) had a documented CRNM bleed; all cases were classified as CRNM bleed because of the need for hospitalization. Twenty patients (20 of 186, 11\%) had at least one admission due to fever or infection and five patients (5 of 186, $2.7 \%$ ) were reported to have had sepsis (one viral, one due to Streptococcus B) on long-term follow-up. A systematic review of studies conducted between 1966 and 1996 reported an incidence of invasive infection of $2.1 \%$ among 484 patients with ITP, including adults and children. $^{8}$ In comparison, a more recent report including nearly 10,000 adults with ITP estimated the cumulative incidence of sepsis at $11.1 \%$ in splenectomized versus $10.1 \%$ in non-splenectomized patients. ${ }^{9}$ Age and the number of co-morbidities and longer follow-up of these patients (median of 25 months of total observation in our study $v$ s. 35 months median time from surgery to sepsis in the adult study) were also associated with higher risk of sepsis and might explain the lower frequency seen in our study.

Trend of platelet count post-surgery in the first 30 days and up to 4 years post-surgery is shown in Figure 1. The peak platelet count after surgery was $549 \times 10^{9} / \mathrm{L}$ (range: 5 $1,944 \times 10^{9} / \mathrm{L}$ ) at a median of 7 days (range: 1-35 days). The median platelet count between $30-60$ days postsurgery was $337 \times 10^{9} / \mathrm{L} \quad\left(25^{\text {th }}-75^{\text {th }}\right.$ percentile: 128 $\left.493 \times 10^{9} / \mathrm{L}\right)$. Generalized estimating equations showed a non-statistically significant decline in platelet count over time of $0.56 \times 10^{9} / \mathrm{L}$ per month, $95 \% \mathrm{CI}: 1.15-0.04, P=0.08$, after the first month post-splenectomy.

Frequency of CR/R/NR is shown in Figure 2. Overall, $93 \%$ of patients showed CR/R; this frequency is slightly higher than that reported among adult patients ${ }^{10}$ and might be explained by the high frequency of splenectomy performed before reaching chronic ITP status $(26 \%)$ and by a relatively low number of patients who received second-line therapy (51 of 239, 21\%). In addition, we observed a significant difference on the median time to surgery from ITP diagnosis before and after 2009 (median time: 1.7 years, $25^{\text {th }}-75^{\text {th }}$ percentile: $0.9-3.1$ years, before and including 2009 , and 3.2 years, $25^{\text {th }}-75^{\text {th }}$ percentile: 
2.0-4.3 years after 2009) when standardized definitions for chronic and persistent ITP were published. ${ }^{4}$ Importantly, the higher frequency of bleeding documented as the response category decreases (Figure 2), suggesting that the classification used in this study approximates clinically relevant outcomes.

Refractoriness was $1.7 \%$ (4 of 239) among all children and $2.6 \%$ (4 of 152 ) in children with $\geq 1$ year of followup. These estimates of refractoriness are slightly lower than those for adult patients, ${ }^{5}$ as we did not take into account administration of platelet-enhancing therapy on follow-up since administration of platelet-enhancing therapy at the time of each platelet count was not reported in the Registry.

Results of simple logistic regression are shown in Table 1. In multivariable analysis, both age at the time of surgery and average platelet count on the first 30 days postsplenectomy predicted CR/R. For every year increase in age at the time of surgery, the odds of CR/R increased by $1.18(95 \% \mathrm{CI}: 1.02-1.40)$ and for every $10 \times 10^{9} / \mathrm{L}$ increase in the average platelet count in the first month, the odds of response increased by 1.09 (95\% CI: 1.05-1.16). In terms of model performance, the optimism-corrected area under the ROC curve was estimated as 0.87 .
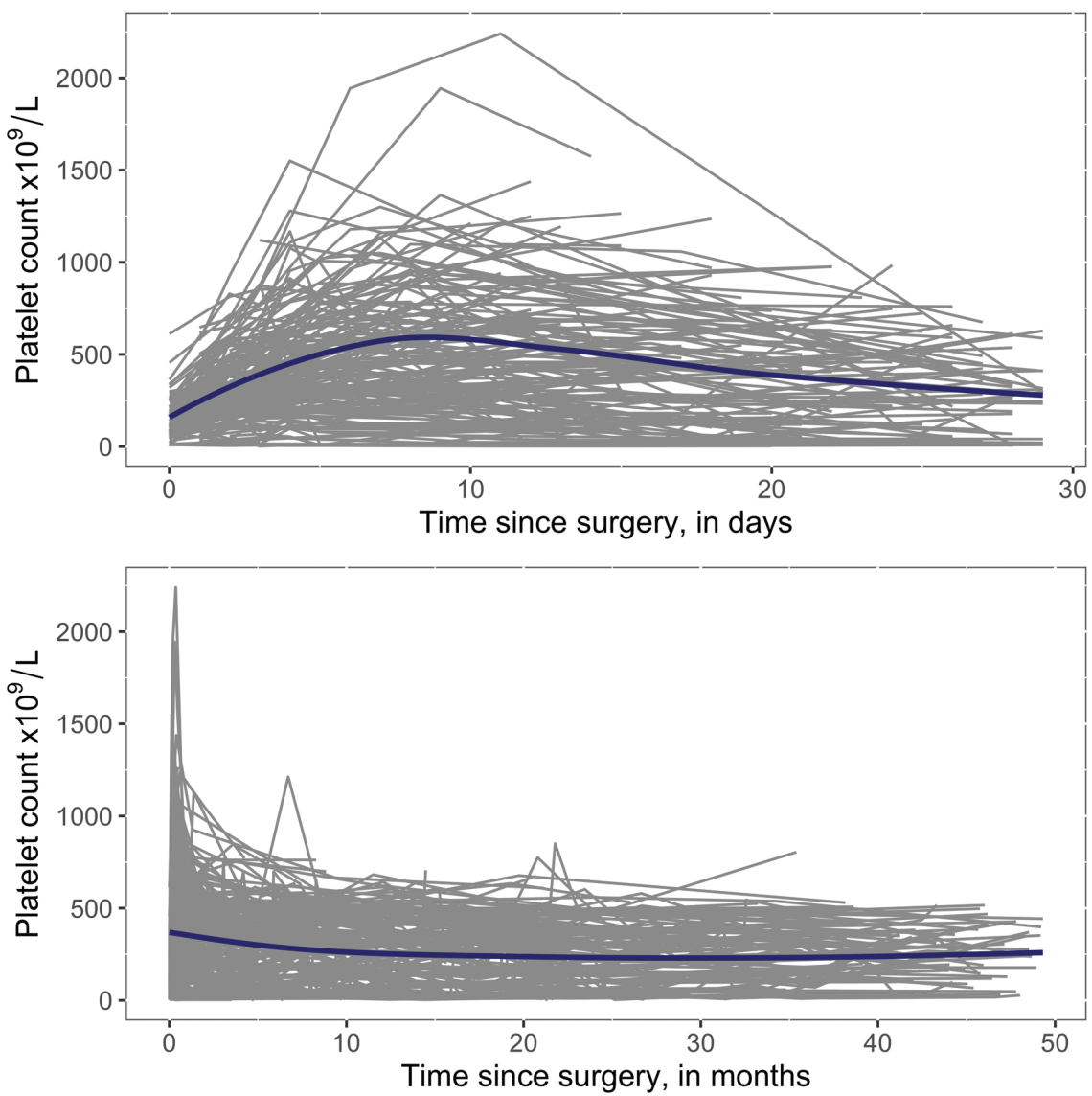

Figure 1. Spaghetti plot showing platelet counts over time. Spaghetti plots of platelet count for each individual over time after splenectomy. The upper panel shows platelet counts in the first 30 days and the lower pane shows platelet counts in the first 50 months of follow-up. The blue solid line represents the regression line (loess).

Table 1. Simple logistic regression results for complete response or response to splenectomy in primary immune thrombocytopenia (ITP).

\begin{tabular}{|c|c|c|}
\hline Predictor & OR (95\%CI) & $P$ \\
\hline Sex (male vs. female) & $0.94(0.30-3.05)$ & 0.92 \\
\hline Age at time of ITP diagnosis, years & $1.12(0.99-1.29)$ & 0.09 \\
\hline Age at time of surgery, years & $1.15(1.01-1.33)$ & 0.04 \\
\hline Time from diagnosis to surgery, years & $1.05(0.86-1.41)$ & 0.71 \\
\hline N. of previous treatment modalities & $0.70(0.41-1.24)$ & 0.21 \\
\hline Second-line therapy (yes vs. no) & $0.30(0.09-0.97)$ & 0.04 \\
\hline Steroids as part of initial therapy (yes $v$ s. no) & $1.02(0.05-5.83)$ & 0.99 \\
\hline IVIG as part of initial therapy (yes vs. no) & $0.59(0.03-3.25)$ & 0.99 \\
\hline Platelet count immediately before splenectomy* & $1.01(1.01-1.03)$ & 0.01 \\
\hline Average platelet count in first 30 days post-splenectomy* & $1.09(1.04-1.15)$ & $<0.001$ \\
\hline Any platelet count $<100 \times 10^{9} / \mathrm{L}$ in first 30 days post-splenectomy (no $v s$. yes) & $26.32(6.62-176.30)$ & $<0.001$ \\
\hline Peak platelet count in first 30 days post-splenectomy* & $1.03(1.01-1.06)$ & 0.009 \\
\hline
\end{tabular}

OR: odds ratio; CI: confidence interval; N: number; IVIG: intravenous immunoglobulin. *For every 10x10\%/L increase. 


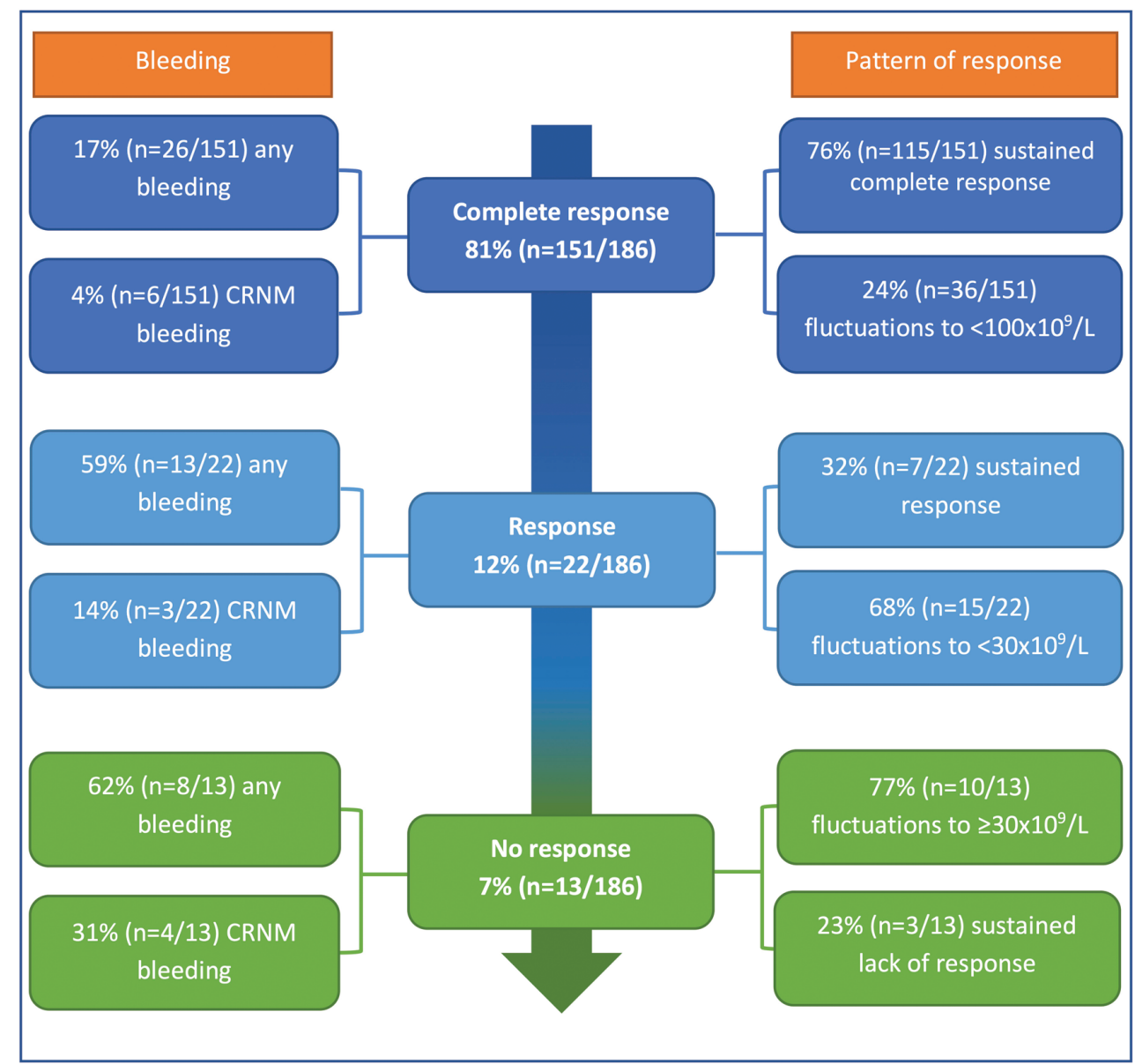

Figure 2. Frequency of response and bleeding in primary immune thrombocytopenia. Center column shows the overall response category and percentage of patients in each of them. Left column shows the frequency of bleeding and clinically relevant non-major (CRNM) bleeding according to response category. Right column shows the frequency of patterns of response in each response category depending on whether the response was sustained or there were fluctuations over time. n: number.

Older age at the time of diagnosis was reported to be an independent predictor of CR in the previous analysis of this Registry ${ }^{3}$ and age at the time of diagnosis was highly correlated with age at the time of splenectomy in the present study. Younger age is considered a predictor of CR/R in adult patients with ITP undergoing splenectomy, ${ }^{11,12}$ perhaps indicating that there might be a similar underlying mechanism among teenagers and young adults. Older age is also a predictor of chronic ITP in pediatrics ${ }^{13}$ further indicating a distinct pathophysiology in ITP affecting teenagers and young adults. It is also possible that patients who undergo splenectomy at a young age are also more refractory to treatment.

Higher platelet counts in the first month post-splenectomy was also an independent predictor of both CR/R in this population. Interestingly, higher platelet counts immediately before surgery, not having platelet counts $<100 \times 10^{9} / \mathrm{L}$ in the first 30 days post-splenectomy, and higher peak platelet count in the first 30 days postsplenectomy were predictors of $\mathrm{CR} / \mathrm{R}$ in univariable analysis. Ahmed et al. reported that platelet count at the time of splenectomy was one of the predictors of CR/R in a cohort of 254 patients that included 87 children. ${ }^{14}$ In addition, high platelet count in the first week after splenectomy has been shown to predict response in some studies conducted in adult patients, although results are inconsistent. ${ }^{10} \mathrm{~A}$ more recent study among 174 adults splenectomized for ITP showed that higher pre- and post-operative platelet count were predictors of response in univariable analysis. ${ }^{12}$ Importantly, pre-surgical and early post-surgical platelet counts may be influenced by platelet enhancing therapy administered pre-operatively in addition to the thrombocytosis seen after splenectomy. Taken together, these observations indicate that individuals able to increase and sustain platelet counts in response to an intervention are more likely to show $\mathrm{CR} / \mathrm{R}$ in the long term.

The present study has some limitations. For example, biases such as selection bias and information bias of an intercontinental registry cannot be ruled out. However, one-third of the patients included in the Registry come from a systematic search at a single center (SickKids) which could help, in part, overcome the issue of selection bias. In addition, the median follow-up time was 25 months, despite the fact that the registry has been open for two decades. Retention can be problematic in registries, particularly when they are voluntary. However, use of registry data can be beneficial as registries are also characterized by strong external validity in view of the heterogeneous populations included, thus better reflecting clinical practice. ${ }^{15}$ In addition, the differential diagnosis in terms of primary or secondary ITP was left to the 
Letters to the Editor

decision of the treating physician, and therefore misclassification cannot be ruled out. However, the high firequincy of response found herein suggests misclassificaion is unlikely and may not have impacted the results. Lastly, there was no specific protocol attached to the registry, and care of the patient was left to the discretion of the clinician, thus reflecting general practice.

In conclusion, splenectomy is a very effective approach for the management of selected children with primary ITP as evidenced by platelet count recovery. Although low, the risk of adverse events both peri-operative and in the long-term should be carefully considered when inticaring this therapeutic modality. The only predictor of response available prior to indication of splenectomy is patient age; the older the patient, the higher the odds of response.

Maria L. Avila, ${ }^{1,2}$ Sour Amiri, ${ }^{1}$ Eleanor Pullenayegum, ${ }^{2}$ Victor Blanchette, ${ }^{1}$ Paul Imbach ${ }^{3}$ and Thomas Kühne on behalf of the Intercontinental Cooperative ITP Study Group

${ }^{1}$ Department of Pediatrics, University of Toronto, Division of Hematology/Oncology, The Hospital for Sick Children, Toronto, Ontario, Canada; ${ }^{2}$ Child Health Evaluative Sciences, The Hospital for Sick Children, Toronto, Ontario, Canada; ${ }^{3}$ Medical Faculty, University of Basel, Switzerland and ${ }^{4}$ UKBB Universitäts-Kinderspital, Oncology/Hematology, Basel, Switzerland

Correspondence:

THOMAS KUEHNE - thomas.kuehne@ukbb.ch

doi:10.3324/haematol.2019.236737

References

1. Cooper N, Bussed J. The pathogenesis of immune thrombocytopaenic purpura. Br J Haematol. 2006;133(4):364-374.

2. Rodeghiero F. A critical appraisal of the evidence for the role of splenectomy in adults and children with ITP. Br J Haematol. 2018;181(2):183-195.

3. Kühne T, Blanchette V, Buchanan GR, et al. Splenectomy in children with idiopathic thrombocytopenic purpura: a prospective study of 134 children from the Intercontinental Childhood ITP Study Group. Pediatr Blood Cancer. 2007;49(6):829-834.
4. Rodeghiero F, Stasi R, Gernsheimer T, et al. Standardization of terninology, definitions and outcome criteria in immune thrombocytopenic purpura of adults and children: report from an international working group. Blood. 2009;113(11):2386-2393.

5. Rodeghiero F, Rugger M. ITP and international guidelines: what do we know, what do we need? Prose Med. 2014;43(4 Pt 2):e61-67.

6. Kaatz S, Ahmad D, Spyropoulos AC, Schulman S; the Subcommittee on Control of Anticoagulation. Definition of clinically relevant nonmajor bleeding in studies of anticoagulants in atrial fibrillation and venous thromboembolic disease in non-surgical patients: communication from the SSC of the ISTH. J Thromb Haemost. 2015; 13(11):2119-2126.

7. Schulman S, Kearon C; the Subcommittee on Control of Anticoagulation of the Scientific and Standardization Committee of the International Society on Thrombosis and Haemostasis. Definition of major bleeding in clinical investigations of antihemostatic medicinal products in nonsurgical patients: definitions of major bleeding in clinical studies. J Thromb Haemost. 2005;3(4):692694.

8. Bisharat N, Omani H, Lavi I, Raze R. Risk of infection and death among post-splenectomy patients. J Infect. 2001;43(3):182-186.

9. Boyle S, White RH, Bronson A, Win T. Splenectomy and the incifence of venous thromboembolism and sepsis in patients with immune thrombocytopenia. Blood. 2013;121(23):4782-4790.

10. Chaturvedi S, Arnold DM, McCrae KR. Splenectomy for immune thrombocytopenia: down but not out. Blood. 2018;131(11):11721182 .

11. Kojouri K. Splenectomy for adult patients with idiopathic thrombocytopenic purpura: a systematic review to assess long-term platelet count responses, prediction of response, and surgical complications. Blood. 2004;104(9):2623-2634.

12. Guan Y, Wang S, Xe F, et al. Long-term results of splenectomy in adult chronic immune thrombocytopenia. Eur J Haematol. 2017; 98(3):235-241.

13. Heitink-Polle KMJ, Nijsten J, Boonacker CWB, de Haas M, Bruin MCA. Clinical and laboratory predictors of chronic immune throwbocytopenia in children: a systematic review and meta-analysis. Blood. 2014;124(22):3295-3307.

14. Ahmed R, Devasia AJ, Viswabandya A, et al. Long-term outcome following splenectomy for chronic and persistent immune thrombocytopenia (ITP) in adults and children: splenectomy in ITP. Ann Hematol. 2016;95(9):1429-1434.

15. Gliklich R, Breyer N, Leave M, eds. Registries for evaluating patient outcomes: a user's guide. 3rd ed. Rockville (MD); Agency for Healthcare Research and Quality (US); 2014.

haematological | 2020; 105(11)

2685 